\title{
Carnets
}

Revue électronique d'études françaises de l'APEF

Deuxième série - 4 | 2015

Regards sur Camus

\section{La pensée politique du dernier Camus}

Jean-Baptiste Dussert

\section{(2) OpenEdition}

\section{Journals}

Édition électronique

URL : http://journals.openedition.org/carnets/1548

DOI : $10.4000 /$ carnets. 1548

ISSN : 1646-7698

Éditeur

APEF

\section{Référence électronique}

Jean-Baptiste Dussert, «La pensée politique du dernier Camus », Carnets [En ligne], Deuxième série 4 | 2015, mis en ligne le 30 mai 2015, consulté le 21 avril 2019. URL : http://journals.openedition.org/ carnets/1548; DOl : 10.4000/carnets. 1548

Ce document a été généré automatiquement le 21 avril 2019.

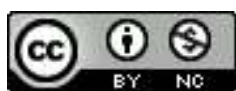

Carnets est mis à disposition selon les termes de la licence Creative Commons - Atribution - Pas d'utilisation commerciale 4.0 International. 


\title{
La pensée politique du dernier Camus
}

\author{
Jean-Baptiste Dussert
}

1 Permettez-moi, pour commencer, une note personnelle. Bien que, d'ordinaire, je ne manque pas, chaque année, de me ruer dans la première librairie venue pour y acheter les œuvres du glorieux lauréat du prix Nobel de littérature, je me suis toujours défié de cette distinction. Elle m'apparaît, de la part de son créateur - l'inventeur de la dynamite, comme chacun sait -, telle une tentative de rédemption désespérée. Dans le domaine des sciences, cet honneur ne souffre guère de critique, car il ne fait souvent qu'honorer un progrès objectif et salutaire de la connaissance humaine. Dans le domaine politique, c'est tout le contraire, car combien de dirigeants, auréolés de cette reconnaissance, n'apparaissent-ils pas, après coup, comme ayant été d'une lâcheté ou d'un machiavélisme édifiant? Mais revenons aux lettres. Quoique l'Académie suédoise soit souvent juste dans ses choix d'écrivains, je me méfie qu'on célèbre une œuvre - dont une bonne part de l'essence stylistique a d'ordinaire été perdue, puisqu'elle a souvent été lue dans une traduction - au seul motif qu'elle sert l'Humanité et témoigne d'un puissant idéal. À mes yeux, la finalité de la poésie, d'un roman ou d'une pièce de théâtre n'est certainement pas d'édifier, de guider ou de soigner. C'est pourquoi je me demande, Albert Camus n'ayant pratiquement rien publié après sa consécration, si les honneurs qui lui furent rendus en 1957 ne nous éloignent pas, un peu plus, de la vérité de son être et de son œuvre; les mêmes considérations vaudraient pour la velléité qu'on a eue, en 2009, de le faire entrer au Panthéon. Cette manière de statufier Camus nous renvoie de lui une image lisse, univoque, bien éloignée, ce me semble, de son véritable tempérament. Sa mort brutale et prématurée, à l'âge de 47 ans, contribue aussi à cette légende qui est assez trompeuse. Qu'il ait été une conscience morale pour son époque et le soit devenu pour la nôtre, nul ne le contestera. Mais ne faut-il pas l'étudier avec plus de circonspection et considérer qu'au moment de sa mort, il était à la veille de devoir faire des choix cruels qui auraient sensiblement modifié l'image que nous avons à présent de lui ? Je vais donc simplement m'efforcer de parler du Camus politique au sens de son rapport à la cité, de son engagement et de sa responsabilité tels qu'ils évoluèrent à la fin de sa vie. 
2 L'image qu'on se forme d'Albert Camus était déjà caricaturale de son temps. Ce manque de perspicacité se retrouvait, d'une part, chez ses panégyristes, qui, éblouis par son style et la justesse de ses intuitions politiques, ne considérèrent pas avec suffisamment de sévérité les réelles faiblesses culturelles de L'homme révolté (1952). Je parle bien de « faiblesse culturelle ». Ce qui est en cause, dans ce livre comme dans d'autres, ce n'est pas le talent ou la puissance intellectuelle de Camus, que tout le monde reconnaît, mais la superficialité de son approche philosophique, que relevait judicieusement Sartre, légèreté qui explique aussi sa plus grande popularité dans l'opinion contemporaine. C'est de la philosophie, certes, mais vulgarisée. Est-ce à dire que ses détracteurs furent, d'autre part, plus justes? Si l'on tient compte de la précision des critiques, qui les rendit d'autant plus acerbes, cela va sans dire. Mais, en termes d'impartialité, leurs attaques furent tout aussi excessives que les louanges adverses.

3 J'observe que les censeurs d'Albert Camus usaient toujours, à son égard, de la même expression de « belle âme ». Attardons-nous un instant sur celle-ci. On la trouve dès 1947, dans un article de Claude Morgan, dans Les lettres françaises, intitulé « Les belles âmes sont impuissantes ", dirigée contre La peste (1947); puis, à la suite de la publication de L'homme révolté (1952), dans un article de Pierre Hervé, "La révolte camuse », publié dans La nouvelle critique; enfin, dans la série d'écrits que Francis Jeanson fit paraître dans Les temps modernes et qui provoquèrent la rupture avec Sartre. Toutes ces revues avaient pour point commun d'être étroitement liées au Parti communiste français. Pour mémoire, ce furent Les lettres françaises qui, cette même année 1947, par un violent article contre le livre du dissident et transfuge soviétique Victor Kravtchenko, I chose freedom (1946), fut à l'origine, à Paris, deux ans plus tard, de ce que l'on qualifia de " procès du siècle ». Cette polémique portait rien moins que sur l'existence du goulag, que ce journal contestait. Quant à La nouvelle critique, sous-titrée la " revue du marxisme militant », comptant dans sa rédaction des intellectuels fameux tels que Jean-Toussaint Desanti et Henri Lefebvre, elle était ouvertement dirigée par le comité central et apportait sans réserve son soutien à Staline. Inutile, enfin, de présenter Les temps modernes, la revue de Jean-Paul Sartre; rappelons toutefois que la publication de L'homme révolté coïncida avec un changement brusque de ligne éditoriale qui conduisit, en même temps que la brouille plus personnelle avec Camus, à une rupture brutale et fondamentale avec Merleau-Ponty, que ce dernier relate dans Les aventures de la dialectique (1955). Certaines de ces critiques furent donc sûrement justes, mais il faut chaque fois deviner en filigrane la ligne imposée par Moscou et plus ou moins suivie par les différentes rédactions.

4 L'article de Pierre Hervé illustre parfaitement cette lecture sans nuance. D'emblée, il prend prétexte d'une anecdote: l'exemple de deux hauts-fonctionnaires coloniaux qui, dans la même conversation, auraient dit apprécier Camus et justifié la répression en Afrique Noire (Hervé, 1952 : 66-67). À trois reprises, dans les trente premières lignes de ce papier, il utilise, pour ces deux sinistres personnages, et pour l'auteur de L'étranger, qu'il va jusqu'à considérer comme leur "directeur de conscience", le qualificatif de "belles âmes ». Disons que s'il s'agit d'un qualificatif, il n'est pas laudatif, on l'aura compris. Sur quel point portent réellement ces attaques? Camus est accusé de «malhonnêteté foncière " (Hervé, 1952: 68), entendez «malhonnêteté intellectuelle», parce qu'il ne parle pas, dans son livre, des crimes du colonialisme et de ceux des Etats-Unis, de l'impérialisme, mais uniquement de ceux, que Pierre Hervé conteste, de l'U.R.S.S. et de ses alliés. À ses yeux, l' «honnêteté » tant célébrée de Camus ne dissimule en fait qu'un anticommunisme viscéral. Il ajoute : 
En vérité, Albert Camus est un tacticien, mais un tacticien qui se défend de l'être et prétend se tenir sur le plan de la morale désintéressée. (...) Camus est un froid raisonneur, dont la démarche oblique et les critiques sournoises ne trahissent en rien les sursauts de conscience. (Hervé, $1952: 69,72$ ).

6
amuse », comme dans celui de Francis Jeanson, dans Les temps modernes, «l'âme révoltée » pour L'homme révolté; toujours cette idée que Camus est une âme, c'est-à-dire tout juste rien pour des athées matérialistes. Mais portons notre attention sur ce premier libellé. L'adjectif camus existe bien en français. Peut-être d'origine provençale, clin d'œil à cette région où l'écrivain trouva refuge sur la fin de sa vie, il désigne un "nez court et aplati ", puis, sans doute par une physiognomonie rudimentaire, quelqu'un de «penaud, désappointé » $\left(\mathrm{xv}^{\mathrm{e}} \mathrm{s}\right.$.), « qui a été trompé dans l'attente de quelque chose » (Dictionnaire de l'Académie, 1694) ; enfin, « rendre un homme camus » signifie familièrement « le réduire à ne savoir que dire" (Dictionnaire de l'Académie, 1835). L'idée sous-jacente, avec une allusion à son physique, est donc que Camus, sous des airs de prôner la révolte, demeurerait en retrait, resterait cois face à certains crimes, moins par pleutrerie que par complicité avec l'ordre établi. Jeanson écrit : « Vous baptisez Révolte le consentement, et le voici dédouané. Par la même occasion vous changez l'indifférence en courage, l'inaction en lucidité et la complicité en innocence » (Jeanson, 1952 : 381). Et, aux yeux de ses détracteurs, telle est l'unique raison du succès de ses écrits philosophiques: ils absolvent la bourgeoisie occidentale, comme les deux colons racistes dont nous parlions à l'instant, de tous leurs crimes. Serviteur du système, Camus serait devenu tellement " tabou » ou « sacré » qu'il ne serait plus admis qu'on critique ce « Grand Prêtre de la Morale absolue» (Jeanson, 1952: 383). Passons sur ces jugements qui suintent la jalousie, et tenons-nous en à ce qu'ils révèlent d'un certain rapport à l'idée d'engagement.

Ses adversaires ne lui reprochent pas tant de faire deux poids deux mesures, que d'adopter à l'égard des événements une bienveillante neutralité. C'est le même reproche qui est contenu dans l'expression dont use Jeanson à l'égard des valeurs qu'il professe, "morale de Croix rouge ", c'est-à-dire que l'organisation humanitaire, comme lui selon eux, intervient mais ne prend pas parti. Or c'est précisément ce reproche qui est attaché à la notion de "belle âme ", qui n'est pas une expression anodine, mais est reprise par les marxistes et les existentialistes, fins connaisseurs de la dialectique hégélienne, de $L a$ phénoménologie de l'esprit (1807). Permettez-moi de citer ce passage, un peu long, mais qui permet de préciser le reproche que tous font à Camus :

La conscience vit dans l'angoisse de souiller la splendeur de son intériorité par l'action et l'être-là [durch Handlung und Dasein (Hegel, 1988 : 433)], et pour préserver la pureté de son cœur elle fuit le contact de l'effectivité et persiste dans l'impuissance entêtée, impuissance à renoncer à son Soi affiné jusqu'au suprême degré d'abstraction, à se donner la substantialité, à transformer sa pensée en être et à se confier à la différence absolue. L'objet creux qu'elle crée pour soi-même la remplit donc maintenant de la conscience du vide. Son opération est aspiration nostalgique [sein Tun ist das Sehnen] qui ne fait que se perdre en devenant objet sans essence, et au-delà de cette perte retombant vers soi-même se trouve seulement comme perdue ; - dans cette pureté transparente de ces moments elle devient une malheureuse belle âme [schöne Seele], comme on la nomme, sa lumière s'éteint peu à peu en elle-même, et elle s'évanouit comme une vapeur sans forme qui se dissout dans l'air (Hegel, 1941 : 189). 

1952. Mais Camus savait déjà précisément, en prenant connaissance de cette réponse violente, ce qu'on lui reprochait et de quoi il s'agissait, car, dans L'homme révolté, il avait lui-même parlé des belles âmes. Il qualifie ainsi les meneurs de l'insurrection russe de décembre 1825 (Camus, 1965 : 557-558), pour leur rendre hommage, en opposition au cynisme d'un Netchaiev ${ }^{1}$ (Camus, 1965 : 570). Camus est bien conscient qu'il s'agit d'une expression ironique, que Hegel dénonce avec elle le «mouvement de retraite » par lequel un individu «se crée à [lui-même], dans sa déception, un monde factice où la morale règne seule " (Camus, 1965: 664²). Il utilise même cette expression en interrogeant le statut de la littérature, se demandant si lire un roman consiste ou non à fuir ainsi la réalité.

Il est toutefois évident que si Camus et ses adversaires usent de la même expression, elle n'a pas la même valeur pour eux et témoigne de la manière divergente dont ils conçoivent l'action politique et le rôle de l'intellectuel (Reid, 2007 : passim). En résumé, Hegel mène une critique assez sévère du romantisme d'Iéna, particulièrement de ce mouvement qui consiste, pour le sujet, après qu'il a dévalorisé le monde, à le fuir. L'une de ses principales cibles, l'un des auteurs les plus caractéristiques de cette attitude, est Novalis qui se caractérise par la Sehnsüchtigkeit. Dans ce terme, nous retrouvons die Sehnsucht, «la nostalgie ", car, la conséquence de sa rupture avec la réalité est que sa propre subjectivité se vide à son tour, et que, ne parvenant plus, dès lors, à s'inscrire dans le monde, il en porte un regret mélancolique. Dans la description de la belle âme que nous citions précédemment, c'est absolument cela qui est en cause : les mots sein Tun ist das Sehnen renvoient évidemment à die Sehnsucht. Alors si, lorsqu'il emploie l'expression de «belle âme ", Camus peut avoir une certaine tendresse pour les idées poétiques qui ont mené certains jusqu'au sacrifice suprême, ainsi les décabristes, il ne se satisfait nullement, comme le laissent accroire ses adversaires, d'un retrait où pourrait se tenir l'artiste ou l'intellectuel. Il écrit: «La révolte (...) s'appuie sur le réel pour s'acheminer dans un combat perpétuel vers la vérité. (...) Loin d'être un romantisme, [elle] prend le parti du vrai réalisme. Si elle veut une révolution, elle la veut en faveur de la vie, non contre elle » (Camus, 1965 : 701). Si Camus ne prône pas la retraite de l'intellectuel, il considère qu'il n'est pas si facile de prendre position dans le réel. Et ses réserves vont s'accentuer dans les dernières années de sa vie.

Tour à tour, trois raisons vont le pousser à s'interroger sur son engagement. En premier lieu, la polémique dont il a été l'objet, au moment de la parution de L'homme révolté, a renforcé sa méfiance déjà ancienne à l'égard de l'intelligentsia. Il avait déclaré en 1945 : "Je ne suis pas un philosophe », et en 1949: "Je ne suis pas un intellectuel». Dans La chute (1956), il a encore pris davantage ses distances, fustigeant les «moralistes» et «humanistes professionnels» (Camus, 1962: 1522 et 1544), raillant cette «Europe philosophe » qui a « remplacé le dialogue par le communiqué » (Camus, 1962 : 1498-1499), dénonçant les «grands hommes du moment» (Camus, 196: 1515) qui attendent l'instauration d'un régime policier comme victoire éristique. La défense publique de telle ou telle cause ne trouve pas davantage grâce à ses yeux : "Il faut que quelque chose arrive, voilà l'explication de la plupart des engagements humains » (Camus, 1962 : 1494), écrit-il. On m'objectera que c'est Jean-Baptiste Clamens qui parle, mais, à peu de choses près, Camus exprime les mêmes opinions dans la conférence qu'il donna à Upsal, le 14 décembre 1957 : «Tout artiste aujourd'hui est embarqué dans la galère de son temps. Il doit s'y résigner, même s'il juge que cette galère sent le hareng, que les gardes-chiourme 
y sont vraiment trop nombreux et que, de surcroit, le cap est mal pris. Nous sommes en pleine mer » (Camus, 1965 : 1079). Cela signifie à la fois que l'engagement est subi par tous et qu'il ne place pas l'intellectuel en position de supériorité. En second lieu, la consécration de son œuvre par le prix Nobel l'a honoré, ému bien sûr, mais aussi profondément affecté psychologiquement, comme en témoigne son journal intime. Non seulement ces honneurs épuisent sa veine créative, ce qui est une conséquence fréquente - comment poursuivre une œuvre qui a déjà été consacrée ? -, mais ils le contraignent à avoir un rôle public auquel il souhaitait se soustraire, à prendre une posture simpliste.

11 En troisième lieu, l'accélération des événements en Algérie fait qu'il s'interroge de plus en plus sur la place à tenir, d'autant que sa situation est exceptionnelle. C'est la principale raison de sa retraite. Dans une lettre hélas non datée, il écrivait : «J'ai décidé, il y a plus d'un an, après avoir reconnu ce qui me séparait irrémédiablement de la gauche comme de la droite sur la question algérienne, de ne plus m'associer à aucune campagne publique sur ce sujet» (Camus, 1989: 251). En l'occurrence, il ne dit pas seulement qu'il ne s'associera plus à aucune pétition, qu'il refusera toute démarche collective parce qu'elle suppose un amalgame équivoque, mais fait l'aveu, à demi-mot, de l'isolement dont il est de plus en plus victime. Pourquoi ? D'une part, il ne peut s'associer avec la droite qui revendique l'Algérie française. Il porte un regard sévère sur les abus du colonialisme, et n'aurait certainement pu se retrouver dans la double évolution qui va la mener, soit à l'abandon de ce territoire, suivant le reniement du général de Gaulle, soit au jusqu'auboutisme de l'O.A.S. D'autre part, la gauche est aveuglée par une lecture purement idéologique et géopolitique, n'envisageant ce conflit que comme la confrontation des blocs et l'aspiration d'un peuple à l'indépendance, ne voyant pas en quoi elle est une guerre civile, une tragédie. Le 5 mars 1958, il a rencontré le général de Gaulle et comprend probablement que l'ancien chef de la Résistance est convaincu de l'impossibilité de trouver une solution pour l'Algérie. En dépit du fameux discours du 4 juin : « Je vous ai compris ! ", il écrit le 29 juillet : « Le matin l'Algérie m'obsède. Trop tard, trop tard... Ma terre perdue, je ne vaudrais plus rien» (Camus, 1989: 251). Il est à la fois convaincu, même s'il ne se l'avoue pas, de la catastrophe à venir, et de la difficulté qu'il éprouverait à y survivre. Mais ce n'est pas un hasard si, le 30 septembre, il acquiert la maison de Lourmarin. La Provence est le seul endroit de métropole où il puisse espérer se réfugier, retrouver un semblant du parfum et des lumières de son pays natal.

Pour conclure, prêtons-nous au jeu de l'uchronie. Qu'aurait fait Albert Camus s'il n'était pas mort le 4 janvier 1960 ? J'espère que ce que je vais dire ne heurtera personne, mais je pense que sa mort prématurée, en même temps qu'elle a servi sa légende, l'a sauvé d'événements qu'il n'était pas possible d'affronter convenablement. Je ne dis pas qu'il y aurait eu compromission, mais des atermoiements qui auraient altéré l'image assez idéale que nous avons de lui. Aurait-il rejoint la droite ? Je ne le pense pas, pour les raisons que je viens d'évoquer. Toutefois, il ne se sentait déjà plus solidaire de la gauche. En septembre 1959, il notait : "Cette gauche dont je fais partie, malgré moi et malgré elle » (Camus, 1989 : 273). Son isolement n'aurait fait que s'accroître lorsque, huit mois après sa disparition, fut lancé le manifeste des 121 (6 septembre 1960). Je pense que sa position aurait été assez semblable à celle de Germaine Tillon, qu'il avait rencontrée régulièrement, peu de temps avant sa mort, et qui, après l'indépendance, se mobilisa, sur le plan humanitaire, pour continuer de venir en aide au peuple algérien. Il avait commencé d'agir de la sorte, en écrivant personnellement à différents responsables politiques, par exemple, pour obtenir des amnisties. Ce travail discret pour apaiser les 
souffrances de cette guerre, cet activisme se développa particulièrement dans les derniers mois de sa vie. Il se serait donc efforcé, par-delà tout engagement politique, de venir en aide à toute cette population, arabes et pieds-noirs, que les événements plongeaient dans la souffrance. Il aurait sans doute définitivement abandonné la politique pour l'humanitaire, pratique plus conforme à sa vision de l'humanisme. C'est pour cela qu'il avait défini l'engagement dans les termes que nous avons énoncés précédemment, montrant que celui-ci n'était pas un choix, mais que les événements s'imposent à vous. On voit bien, dès lors, combien Camus n'était pas cette "belle âme ", au sens hégélien du terme, car il ne pouvait nullement se satisfaire de l'indifférence. Contrairement à ses anciens amis, devenus ses adversaires, tous ses choix, toutes ses paroles l'engageaient du plus profond de son être. Il n'était pas une belle âme, mais une âme déchirée.

\section{BIBLIOGRAPHIE}

CAmus, Albert (1962). La chute, in Théâtre, récits, nouvelles. Paris : Gallimard, « Bibliothèque de la Pléiade ».

CAMUS, Albert (1965). L'homme révolté, in Essais. Paris : Gallimard, « Bibliothèque de la Pléiade ». CAMUS, Albert (1989). Carnets III. Paris : Gallimard.

HEGEL, G. W. F. (1988). Phänomenologie des Geistes. Hamburg : Felix Meiner.

HEGEL, G. W. F. (1941). Phénoménologie de l'esprit. Paris : Aubier-Montaigne.

HERVÉ, Pierre (Avril 1952). « La révolte camuse », in Nouvelle critique, pp. 66-76.

MORGAN, Claude (4 juillet 1947). « Les belles âmes sont impuissantes », in Les lettres françaises.

REID, Jeffrey (2007). L'anti-romantique : Hegel contre le romantisme ironique. Laval : Presses de

l'Université de Laval.

\section{NOTES}

1. Faut-il rappeler que l'assassinat de l'étudiant Ivanov (1869), commandité par Netchaiev, inspira à Dostoïevski Les possédés (1871), adapté au théâtre, par Camus, en 1959 ?

2. Cf. Camus, $1965: 543$ et 558. 


\section{RÉSUMÉS}

Tout au long de sa carrière littéraire, Albert Camus fut victime d'une certaine condescendance de la part des philosophes " professionnels ». Celle-ci conduisit à sa fameuse rupture avec Jean-Paul Sartre. Le présent article s'interroge sur l'isolement politique qui frappait l'auteur de L'homme révolté dans les dernières années de sa vie et s'imagine quelle aurait pu être son attitude s'il avait connu l'indépendance de l'Algérie.

Throughout his literary career, Albert Camus was the victim of a certain condescension on the part of "professional" philosophers. This led to his famous break with Jean-Paul Sartre. This article examines the political isolation of the author of The Rebel in the last years of his life and imagine how he would have reacted to the independence of Algeria.

INDEX

Keywords : beautiful soul, revolt, Algeria, engagement, decolonization

Mots-clés : belle âme, révolte, Algérie, engagement, décolonisation

\section{AUTEUR}

\section{JEAN-BAPTISTE DUSSERT}

Un. Paris-Sud

jean-baptiste.dussert[at]u-psud.fr 